\title{
Orbit Design for the Martian Moons Exploration Mission
}

\author{
By Naoko OGawa, ${ }^{1)}$ Yuichi TsudA, ${ }^{1)}$ Yuto TAKeI, ${ }^{2)}$ \\ Hiroka InOUE, ${ }^{1)}$ Shota TAKAHASHI, ${ }^{3)}$ and Yasuhiro KAWAKATSU ${ }^{1)}$ \\ ${ }^{1)}$ Institute of Space and Astronautical Science, JAXA, Sagamihara, Japan \\ ${ }^{2}$ Research and Development Directorate, JAXA, Sagamihara, Japan \\ ${ }^{3)}$ Department of Mechanical Engineering, Keio University, Yokohama, Japan
}

(Received June 30th, 2017)

\begin{abstract}
The Martian Moons eXploration (MMX) mission is now under study by the Japan Aerospace Exploration Agency (JAXA). Its scope includes the world's first landing on one of the Martian moons, collecting samples from the surface, and returning to Earth. This paper describes the orbit design for MMX. Nominal and backup trajectories for launch in 2024 and 2026 are discussed. The Mars orbit insertion (MOI) sequence using 3-impulse maneuvers is introduced. A new scheme, the robust MOI, is also proposed as a contingency to enhance the robustness of the mission sequence. A method to design a robust MOI trajectory and examples are presented.
\end{abstract}

Key Words: Mars, Exploration, Orbit, Sample Return, Phobos, Deimos

\section{Introduction}

The Japan Aerospace Exploration Agency (JAXA) is now planning the Martian Moons eXploration (MMX) mission for launch in the early 2020s. This mission will involve close-up remote sensing and in-situ observation of two Martian moons, Phobos and Deimos, and return samples from Phobos. The mission aims to clarify the origin of the Martian moons using remote sensing and sample return. Determining the origin will be a step toward constraining the behavior of small bodies and clarifying the evolution of the early solar system. It also includes engineering challenges such as the world's first sample return from inside the Martian system. In this article, we will describe the orbit and mission design for this MMX mission.

The spacecraft will be inserted into the Mars orbit and then transferred to the Quasi-Satellite Orbit (QSO) of Phobos. This article focuses on the interplanetary trajectory and Mars orbit insertion/escape. The orbit design and mission design on QSO will be described in other articles. ${ }^{1)}$

\section{Overview of Spacecraft}

Table 1 presents an overview of the proposed spacecraft system specifications derived from system requirements and investigations of the system configuration.

\section{Orbit Design}

\subsection{Launch windows}

The MMX mission includes a planned launch in the 2020s. Launch opportunities that require low energy and short flight time are 2022, 2024, 2026, and 2028. We currently assume the nominal launch in 2024, and the backup in 2026. The arrival at Mars will be one year after the launch. Mars departure opportunities also come every two years (e.g., 2024, 2026, 2028, and 2030). The earliest departure opportunity after the Mars arrival in 2025 will be 2026. In this case, the phase around Mars will be less than one year, which is too short for sufficient ex-
Table 1. System outline draft specification.

\begin{tabular}{|c|c|}
\hline Item & Specification \\
\hline $\begin{array}{l}\text { Propulsion } \\
\text { configuration }\end{array}$ & $\begin{array}{l}\text { Outgoing: chemical propulsion. Re- } \\
\text { turn: chemical propulsion }\end{array}$ \\
\hline $\begin{array}{l}\text { Spacecraft configura- } \\
\text { tion }\end{array}$ & $\begin{array}{l}\text { Propulsion Module / Exploration } \\
\text { Module / Return Module }\end{array}$ \\
\hline Launch & $\begin{array}{l}\text { Rocket: H3-24L, Launch date: Sum- } \\
\text { mer } 2024\end{array}$ \\
\hline Total mission lifetime & +5 years \\
\hline Target mass & $3,500 \mathrm{~kg}$ \\
\hline $\begin{array}{l}\text { Power consumption } \\
\text { Orbital control }\end{array}$ & $\begin{array}{l}\text { Approx. } 900 \mathrm{~W} \\
\text { delta-V: Approx. } 5 \mathrm{~km} / \mathrm{s} \text { (chemical } \\
\text { propulsion) }\end{array}$ \\
\hline
\end{tabular}

ploration. Thus, we assumed a 3-year stay around Mars, with departure in 2028. Return to Earth will occur in 2029.

\subsection{Conditions for launch windows}

We also considered the following conditions for the launch windows:

- $C_{3}$ on Earth departure should be within $18 \mathrm{~km}^{2} / \mathrm{s}^{2}$ (TBD).

- Declination of launch asymptote (DLA) should be within \pm 30 degrees constrained by the launch site (Tanegashima Space Center) and the vehicle capabilities.

- Time of flight should be within one Earth year.

- More than 2 weeks in succession should be available as the launch window.

\subsection{Interplanetary trajectory}

Considering the assessment results discussed above, we assumed the nominal launch date as 2024 and the return date as 2029, and designed trans-Mars orbits. Note that these preliminary results presuming ballistic flights are not optimized, and no multibody dynamics, perturbations, or trajectory correction maneuvers are considered thus far.

Table 2 and Figs. 1, 2 show an interplanetary orbit and its properties.

\subsection{Launch and transfer to Mars}

The spacecraft will be launched from Tanegashima Space Center using an H3 launch vehicle. The rocket will be launched 
Trans. JSASS Aerospace Tech. Japan Vol. 17, No. 3 (2019)

Table 2. Design results of interplanetary trajectories for MMX.

\begin{tabular}{llllll}
\hline & \multicolumn{3}{c}{ Nominal } & \multicolumn{3}{l}{ Backup } \\
\cline { 2 - 6 } & & Earth-Mars & Mars-Earth & Earth-Mars & Mars-Earth \\
\hline \multirow{4}{*}{ Departure } & Date & $2024 / 9 / 11$ & $2028 / 8 / 6$ & $2026 / 10 / 1$ & $2030 / 9 / 10$ \\
\cline { 2 - 6 } & Vinf [km/s] & 3.896 & 2.623 & 3.937 & 2.566 \\
\cline { 2 - 6 } & RA [deg] & 111.570 & 322.966 & 144.541 & 4.117 \\
\cline { 2 - 6 } Arrival & DEC [deg] & 13.277 & -2.309 & 17.009 & -13.512 \\
\hline & Date & $2025 / 8 / 12$ & $2029 / 7 / 12$ & $2027 / 8 / 27$ & $2031 / 8 / 23$ \\
\cline { 2 - 6 } & Vinf $[\mathrm{km} / \mathrm{s}]$ & 2.419 & 3.501 & 2.686 & 4.248 \\
\cline { 2 - 6 } & RA [deg] & 79.503 & 7.836 & 110.144 & 22.828 \\
\cline { 2 - 6 } Time of Flight [days] & DEC [deg] & 7.831 & 22.287 & -10.282 & 37.134 \\
\hline Inclination Change [deg] & 10.359 & 340 & 328 & 347 \\
\hline MOI1/MOE3 DV [km/s] & 0.653 & 0.750 & 0.781 & 0.722 \\
\hline MOI2/MOE2 DV [km/s] & 0.075 & 0.070 & 0.078 & 0.069 \\
\hline MOI3/MOE1 DV [km/s] & 0.786 & 0.786 & 0.786 & 0.786 \\
\hline DV Total [km/s] & 1.514 & 1.605 & 1.645 & 1.593 \\
\hline
\end{tabular}

toward the east with an azimuth angle of 90 degrees, and injected into a coasting orbit at $300-\mathrm{km}$ altitude after separation of the first stage. Either long or short coasting will be adopted considering visible pass conditions. The spacecraft will be separated after the second engine ignition.

The Mars Transfer Phase lasts from the end of the Early Orbit Phase until around one month prior to MOI. Regular health checks of the PI instruments will be planned. The interplanetary cruise will be a direct transfer orbit from Earth to Mars. No gravity assist is planned during this phase. The spacecraft will be guided toward the MOI targeting point around Mars using several trajectory correction maneuvers (TCMs), as well as precise orbit determination including ranging, twoway Doppler, and delta-differential one-way ranging (DDOR). The cruise phase will last approximately 11 months according to current candidate profiles. The nominal arrival time will be around July 2025.

\subsection{Mars orbit insertion and transfer to Phobos co-orbit}

The final target orbit in the Martian system is the Phobos co-orbit. Because its inclination is around zero degrees with respect to the Mars equator, it is difficult to insert the spacecraft directly from interplanetary space. The inclination just after insertion is bounded by the declination of incoming hyperbolic excess velocity; thus the direct insertion into the orbit on the equator requires almost zero-degree declination for the approach, which is not feasible.

In order to avoid such difficulty, we adopted three-staged Mars orbit insertion (MOI) where the spacecraft will first be injected into an ellipsoidal orbit, then into an intermediate orbit, and finally into the Phobos co-orbit.

In the first MOI (MOI1), the spacecraft will be injected into an ellipsoidal orbit with an apoapsis of $40 \mathrm{Rm}$ (TBD, Rm is the Mars radius) and a periapsis altitude of $500 \mathrm{~km}$ (TBD). There is a degree of freedom for the B-plane phase angle $\theta$ in MOI1, which allows us to choose the inclination or the argument of periapsis $\omega$ of the first ellipsoidal orbit. If we set the argument of periapsis to around 0 or 180 degrees, the apoapsis will be placed within the equatorial plane. This means that we can change the inclination at the apoapsis in the following MOI2 and MOI3 maneuver sequences, leading to drastic reduction of fuel consumption. When $\omega$ is 0 or 180 degrees, $\theta$ can be expressed using a half-cone angle $\rho=\arccos (1 / e)$ between incoming and outgoing asymptotes, incoming asymptote declination $\delta$ and the incoming asymptote vector $\boldsymbol{S} ; \sin \theta=$ $\{\cos \rho \cos \delta\} /\left\{S_{z} \sin \rho\left(S_{x}^{2}+S_{y}^{2}\right)\right\}$. Thus we can choose appropriate $\theta$ to set $\omega$ to 0 or 180 degrees. For example, for the launch in 2024, the B-plane phase angle should be about 7 degrees.

Figure 3 illustrates an example of the geometry in MOI1. The spacecraft will approach from the dawn side of Mars (blue line) and fly toward the dusk side. In this case we will not experience solar or Martian eclipses, although they can occur according to injection conditions. The apoapsis altitude will be fixed later considering the ambiguity of the engine thrust, perturbation by Sun and Mars, the orbital period and operabillity.

After about 3.5 days (TBD), the second orbital maneuver (MOI2) will be executed at the apoapsis, and the periapsis and inclination will be adjusted so that they are the same as those of the Phobos co-orbit. According to the obit determination status, this maneuver may be performed after the second pass over the periapsis.

About 2.5 days later (TBD), the third orbital maneuver (MOI3) will be performed to lower the apoapsis to the Phobos co-orbit. Finally this maneuver places the spacecraft in the Phobos co-orbit. Actually, MOI3 will be divided into several maneuvers during which the Propulsion Module will be jettisoned after the propellant is exhausted, and the remaining delta- $\mathrm{V}$ can be performed by thrusters in the Return Module. The module(s) will be jettisoned in a sufficiently stable Mars orbit to avoid crashing on Mars, in an effort to satisfy planetary protection requirements. The total duration of MOI1, 2 and 3 will be approximately 2 weeks (TBD). Commissioning of some PI instruments will be performed after completion of MOI.

The total amount of delta-Vs for MOI1, 2 and 3 is about $2,000 \mathrm{~m} / \mathrm{s}$ at maximum.

For example, the orbit diagram for MOI1, 2 and 3 for the 2024 launch is presented in Fig. 4.

\subsection{Mars orbit escape}

For Mars Orbit Escape (MOE) before returning Earth, we assume a reversed sequence of MOI. MOE consists of three orbit control maneuvers: MOE1, MOE2, and MOE3. Prior to those maneuvers, the spacecraft is around the Phobos or Deimos coorbit. In MOE1, the apoapsis will be raised to around 40 Mars 

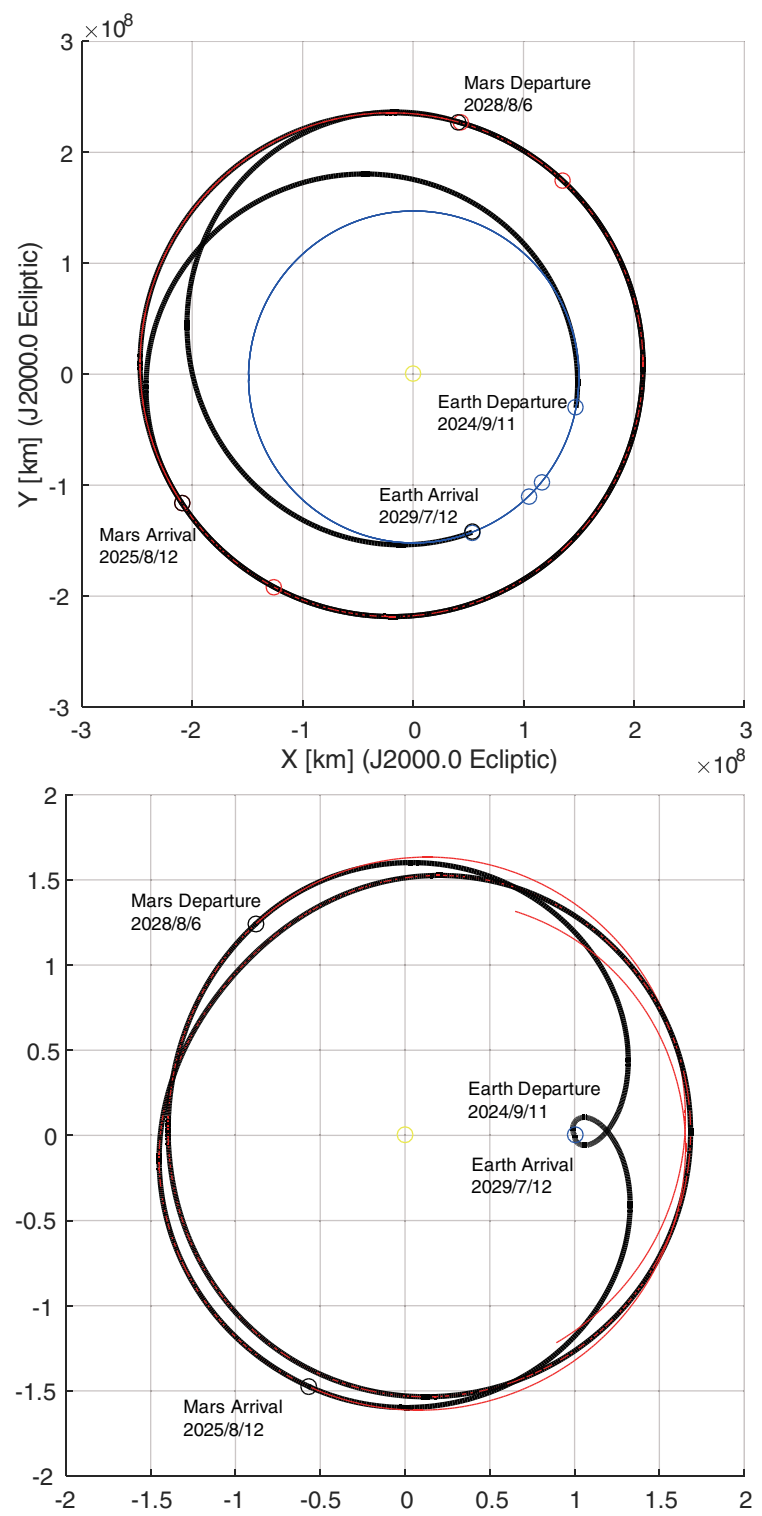

Fig. 1. An example for nominal interplanetary trajectories. Top: J2000 inertial frame. Bottom: Sun-Earth Fixed rotating frame.

radii (TBD). At apoapsis, the subsequent MOE2 maneuver will change the orbit inclination in preparation for escape taking the escape declination into account, and lower the periapsis altitude to $500 \mathrm{~km}$ (TBD). Then MOE3 burn at periapsis will then insert the spacecraft into an interplanetary trajectory, escaping Mars. The total duration of MOE1-3 will be approximately 2 weeks (TBD). The exploration module may be jettisoned after the final scientific observation and before MOE1, depending on the module configuration. The module(s) will be jettisoned in a sufficiently stable Mars orbit to avoid crashing on Mars, in an effort to satisfy planetary protection requirements. The detailed timing of jettison is TBD. The total amount of delta-Vs for MOE1, 2 and 3 is about 2,000 m/s at maximum.

\subsection{Options for rendezvous or flyby of Deimos}

After completion of Phobos observation and sampling, several flybys around Deimos or rendezvous with Deimos will be planned. Actual decisions will depend on the amount of remaining fuel.

For a rendezvous, a Hohman transfer will insert the space-
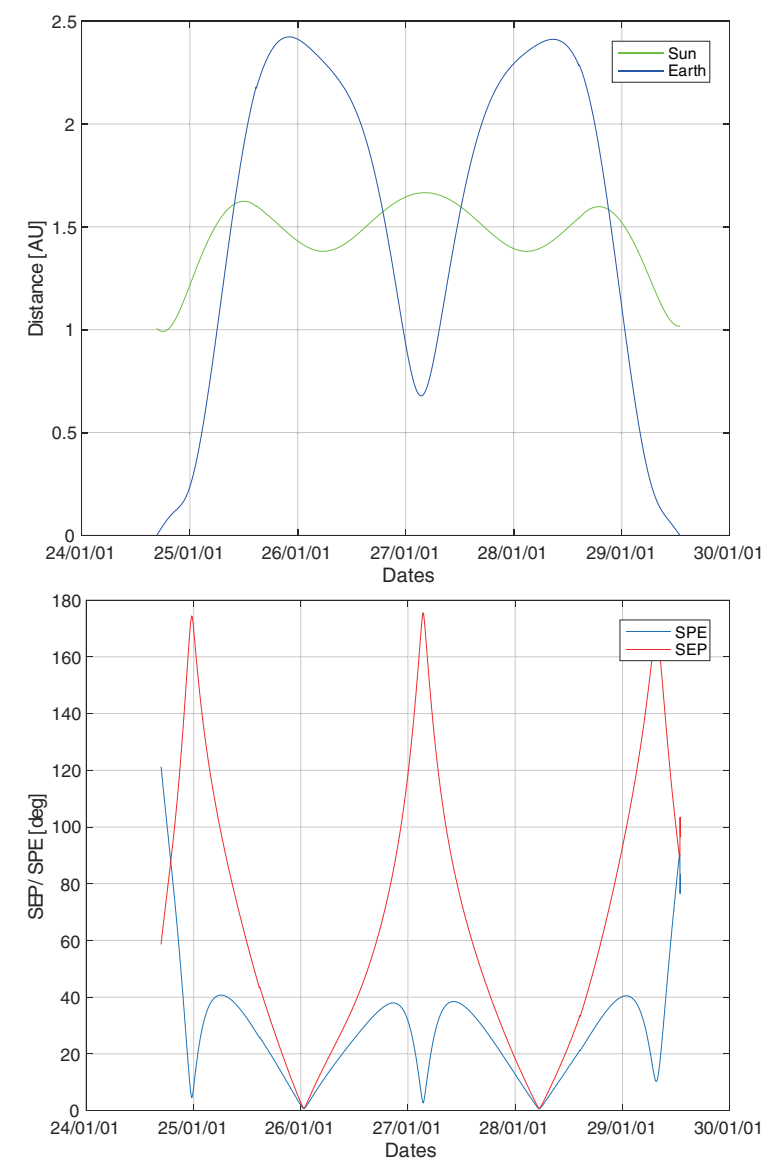

Fig. 2. Sun and Earth distance, Sun-Probe-Earth (SPE) angle, and SunEarth-Probe (SEP) angle for the nominal trajectory.

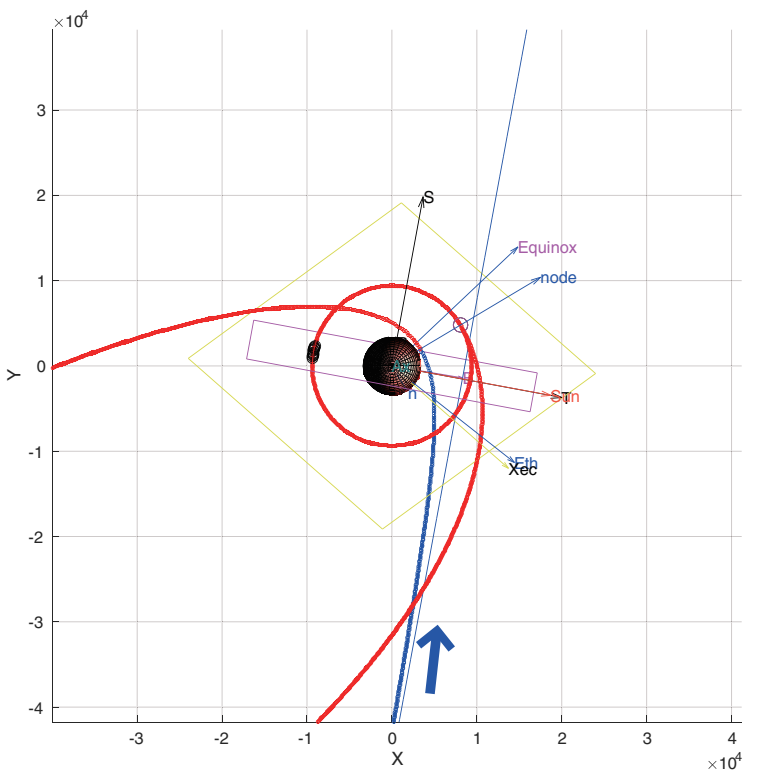

Fig. 3. Example of Mars orbit insertion (X-Y plane: Mars equatorial plane, Z: Martian north).

craft into Deimos-revolution orbit. This requires about $800-\mathrm{m} / \mathrm{s}$ delta- $\mathrm{V}$ at the apoapsis and the periapsis. However, the MOE delta-V from the Deimos QSO is about $300 \mathrm{~m} / \mathrm{s}$ lower than that from the Phobos QSO. Thus the balance is about $500 \mathrm{~m} / \mathrm{s}$ excess compared to the Deimos flyby.

For a flyby, the apoapsis will be raised up to or beyond the 

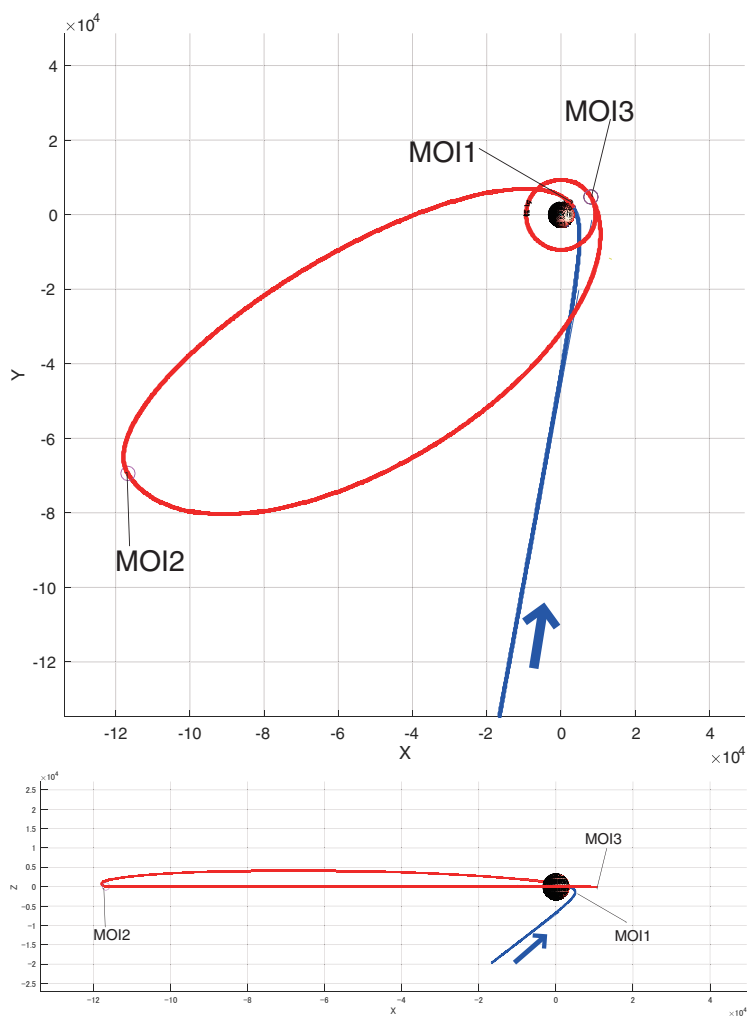

Fig. 4. Orbit diagram for MOI1-3.

Deimos orbit so that the spacecraft intersects the Deimos orbit, and orbital resonance will be achieved between the spacecraft and Deimos. The spacecraft will encounter Deimos several times in the flyby orbit. The duration of Deimos flybys or rendezvous is TBD.

\subsection{Deimos flyby option}

The spacecraft will be placed in an ellipsoidal orbit with 40$\mathrm{Rm}$ apoapsis after MOI2 and before MOE2, which will provide opportunities for Deimos flyby at intercept points with the Deimos orbit. Moreover, if the transfer between this ellipsoidal orbit and the Phobos co-orbit is divided into two-stage transfers via an orbit with an intermediate apoapsis, almost no additional delta- $\mathrm{V}$ is needed for adjusting encounter conditions with Deimos.

The encounter period and the relative velocity are the two major parameters in the Deimos flyby. To encounter Deimos periodically, the ratio of their orbital periods should be a simple rational number. Relative velocity will be an input for scientific instruments. These parameters are determined based on the apoapsis altitude.

Figure 5 plots the relationships among apoapsis altitude, orbital period and relative velocity in the Deimos flyby. The upper limit of the orbital period and the relative velocity is derived just after MOI1. The lower limit appears when the apoapsis reaches the Deimos co-orbit.

\subsection{Return to Earth}

The Earth Transfer Phase lasts from the end of MOE until around one month prior to capsule re-entry to Earth. The interplanetary cruise will be a direct transfer orbit from Mars to Earth. No gravity assist is planned during this phase. The spacecraft will be guided toward the capsule separation targeting point around Earth using several TCMs and precise orbit de-

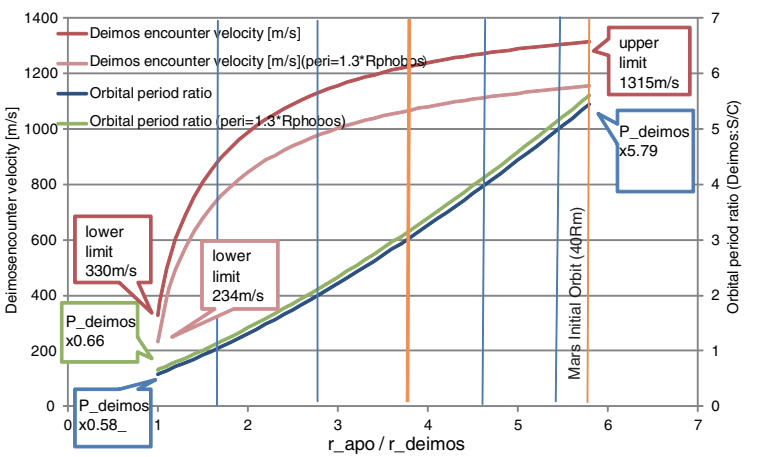

Fig. 5. Apoapsis altitude, orbital period and relative velocity in Deimos flyby.

termination, including ranging, two-way Doppler, and DDOR. The cruise phase will last approximately 11 months, according to candidate profiles.

Near Earth, the spacecraft will target the re-entry interface point. The capsule will be separated from the spacecraft several hours prior to Earth atmosphere re-entry. The capsule will re-enter the Earth atmosphere, descend using a parachute, land on the ground, and be recovered immediately. After capsule separation, the spacecraft will de-orbit from the trajectory targeting the interface point using the chemical propulsion system to escape from Earth's gravity to interplanetary space.

\section{Robust MOI}

\subsection{Concept of robust MOI}

MOI is one of the most critical events in this mission. If the main thruster produces no thrust or insufficient thrust during MOI, the spacecraft cannot be injected into the Mars orbit and will fly away from the Martian system to interplanetary space again. In the MOI sequence of MMX, over 90 percent of the planned thrust is required for capture by Mars' gravity. Akatsuki, the Venus climate orbiter, has confirmed difficulty of orbit insertion and re-encounter with the target after insertion failure.

In MMX, we propose utilization of Mars evolution synchronous orbit (a free return orbit that is also known as an interplanetary parking orbit) to reduce the difficulty of re-encounter. In this scheme, the targeting point on the MOI B-plane is chosen to connect to a Mars evolution synchronous orbit in the event of thrust failure. If the MOI is successful, the spacecraft will be injected into the planned orbit as a nominal sequence. Even if the thruster does not work at all, the spacecraft will go away but then return to Mars. This will drastically enhance the robustness of the mission. Mars evolution synchronous orbits with a period of one Martian year has a degree of freedom for the escape direction from Mars, ${ }^{2)}$ ) which makes it easy to satisfy orbital conditions for both success and failure. Hereafter, this scheme is labeled "robust MOI."

\subsection{How to design robust MOI}

In the robust MOI scheme, there is a constraint that the MOI trajectory must connect to the re-encounter orbit (Mars evolution synchronous orbit) when the MOI thrust is zero. The following is an example of how to design such trajectories roughly using patched conics.

Two trajectories, one just after the MOI failure and the interplanetary re-encounter orbit, can be connected smoothly. Thus, 
the magnitude and the direction of the hyperbolic excess velocity vectors for the two trajectories should be the same. We must match three parameters: the magnitude, the right ascension and the declination of the hyperbolic excess velocity vectors. Fortunately, a Mars evolution synchronous orbit is independent of the magnitude of the hyperbolic excess velocity, and its direction has one degree of freedom in its design. Thus, we can choose two of the three, magnitude and declination for the Mars evolution synchronous orbit, to match those just after the failure. The remaining parameter, the right ascension, depends on the other two parameters. Next, another parameter, the arrival date, is adjusted to match the right ascension. The following is an example of the search sequence:

1. Fix the departure date. Set the arrival date and solve the Lambert problem.

2. From the approaching V-infinity vector to Mars, obtain the appropriate phase angle on the B-plane, $\theta$, in order to set the major axis of the ellipsoidal orbit on the Martian equatorial plane.

3. From the B-plane target derived in the previous procedure, calculate the outgoing velocity vector from Mars in the MOI failure.

4. Fix only the declination of the outgoing vector, and survey the right ascension to connect to the Mars evolution synchronous orbit. Two solutions are obtained: inbound and outbound orbits.

5. Compare the right ascension for the outgoing vector in MOI failure and that for Mars evolution synchronous orbit. If the difference is sufficiently small, it means that the two vectors can be connected; the outgoing orbit can re-encounter Mars, and the robust MOI trajectories can be accomplished. Otherwise, change the arrival date and go back to step 1.

It is noteworthy that the robust MOI trajectory allows the spacecraft to be inserted into the free-return orbit with almost no additional delta- $\mathrm{V}$, if there is no burn at MOI. The spacecraft will almost automatically return to the Mars. If the burn was executed partially in MOI, then the outgoing vector changes and a deep space maneuver will be needed after insertion into the re-encounter orbit (timing should be decided by optimization). The amount of such a maneuver will be several hundred $\mathrm{m} / \mathrm{s}$, which will be made affordable by giving up the Deimos rendezvous. However, whether the MOI succeeds or fails, the delta-V for the robust MOI tends to be more than nominal MOI. It is like insurance for the the MOI failure. An example will be presented in the next section.

An alternate and more analytical approach independent of the target bodies is also under investigation. ${ }^{3) \text { ) }}$

\subsection{Examples}

Table 3 and Fig. 6 present a design example of the robust MOI. In this example, the spacecraft tries MOI on $10 \mathrm{Au}-$ gust 2025 and flies-by Mars at an altitude of $540 \mathrm{~km}$, but reencounters Mars after one Martian year.

This robust MOI orbit is designed to match the case with no MOI thrust. If the thrust is non-zero but not sufficient for MOI, the escape direction will be changed and the spacecraft will not re-encounter Mars. However, an additional deep space maneuver of several hundred $\mathrm{m} / \mathrm{s}$ can force the spacecraft to
Table 3. Example of the trajectory for robust MOI.

\begin{tabular}{llll}
\hline & & Earth-Mars & Mars-Mars \\
\hline \multirow{4}{*}{ Departure } & Date & $2024 / 9 / 11$ & $2025 / 8 / 10$ \\
\cline { 2 - 4 } & Vinf $[\mathrm{km} / \mathrm{s}]$ & 3.895 & 2.420 \\
\cline { 2 - 4 } & RA $[\mathrm{deg}]$ & 111.294 & 162.362 \\
\cline { 2 - 4 } Arrival & DEC $[\mathrm{deg}]$ & 12.753 & 7.968 \\
\hline \multirow{5}{*}{} & Date & $2025 / 8 / 10$ & $2027 / 6 / 28$ \\
\cline { 2 - 4 } & Vinf $[\mathrm{km} / \mathrm{s}]$ & 2.420 & 2.418 \\
\cline { 2 - 4 } & RA $[\mathrm{deg}]$ & 80.307 & 162.418 \\
\cline { 2 - 4 } & DEC $[\mathrm{deg}]$ & 7.968 & 8.013 \\
\hline
\end{tabular}

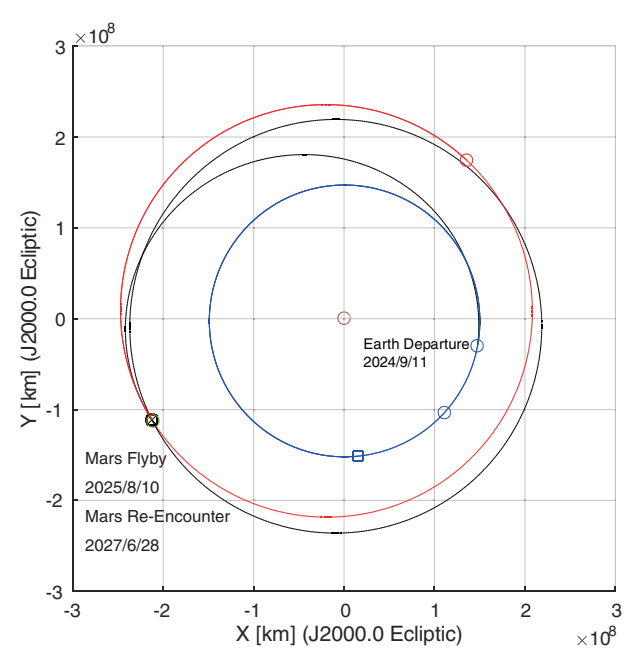

Fig. 6. Example trajectory of the robust MOI.

re-encounter, even if the MOI thrust is 0 to 90 percent of the planned value. We confirmed that each day in the two-week launch window has a robust MOI solution.

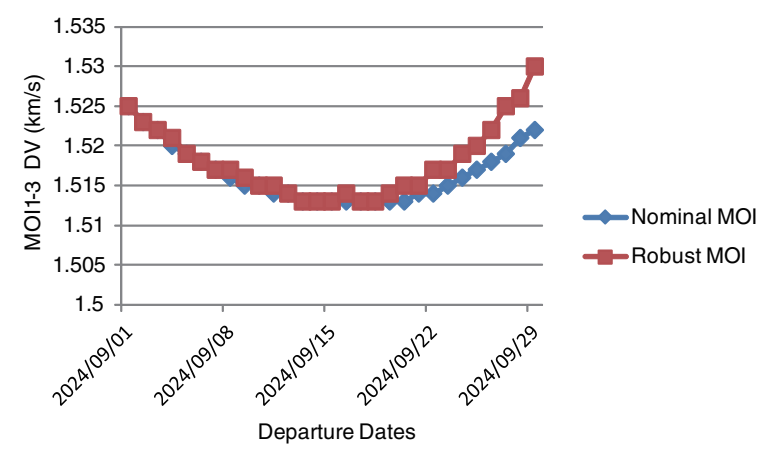

Fig. 7. Comparison of the total delta-V needed for nominal and robust MOI (launch in 2024).

Figures 7 and 8 compare the total delta- $\mathrm{V}$ needed for nominal and robust MOI for launch in 2024 and 2026. The vertical axis indicates the total delta-Vs (MOI1 + MOI2 + MOI3) needed for nominal and robust MOIs. The blue line indicates the minimum delta- $\mathrm{V}$ for the nominal MOI subject to each departure date, where the design variable is the arrival date. The red line denotes the delta- $\mathrm{V}$ for the robust MOI, not always minimized, but designed so as to connect to one-Martian-year re-encounter orbit after the Mars flyby when the thrust fails. The delta-Vs needed for robust and nominal MOIs are almost the same in the 2024 launch, because orbit conditions for the nominal MOIs are close to those for the robust MOIs. Thus 2024 is a good launch opprtunity to realize the robust MOI with almost no system impacts. However, the delta-Vs needed for robust MOIs are much 


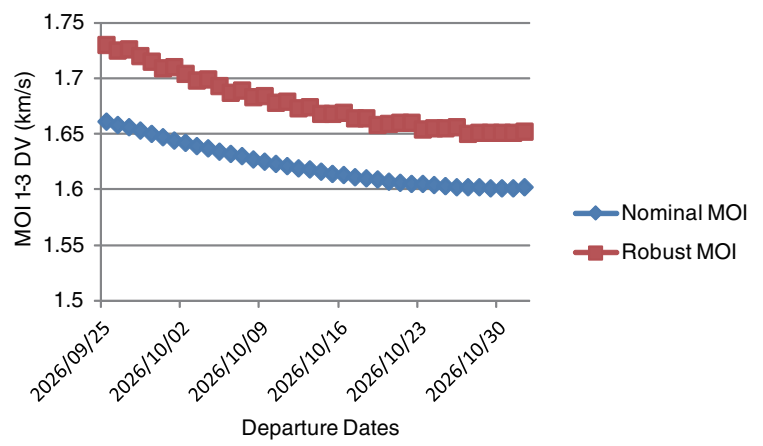

Fig. 8. Comparison of the total delta- $\mathrm{V}$ needed for nominal and robust MOI (launch in 2026)

larger than those for nominal MOIs in the case of 2026 launch. In that case, additional delta-Vs of about 50 to $70 \mathrm{~m} / \mathrm{s}$ are required for the robust MOI. This is an important finding for the mission design of the MMX.

\section{Summary}

We have described a preliminary trajectory plan for the MMX mission. Feasible interplanetary transfer trajectories and orbit insertion including robust MOI methods were proposed considering mission requirements. In future works, the operation analysis, consideration of planetary protection, and contingency and backup plans will be discussed.

\section{References}

1) Ikeda, H., Mitani, S., Mimasu, Y., Ono, G., Nigo, K. and Kawakatsu, Y.: Orbital Operations Strategy in the Vicinity of Phobos, Proceedings of the 26th International Symposium on Space Flight Dynamics / 31 st International Symposium on Space Technology and Science, Matsuyama, 2017, ISSFD-2017-008 / ISTS-2017-d-008. .

2) Ogawa, N., Mimasu, Y., Tanaka, K., Yamaguchi, T., Fujita, K., Narita, S. and Kawaguchi, J.: Earth Revolution Synchronous Orbits and AeroGravity Assists to Enhance Capabilities for Interplanetary Missions by Sub-Payload Spacecraft, Proceedings of 13th International Space Conference of Pacific-basin Societies, Kyoto, 2012, C1-9.

3) Takahashi, S.: Method of Robust Orbit Insertion and its Application to MMX Mission, Proceedings of the 26th International Symposium on Space Flight Dynamics / 31st International Symposium on Space Technology and Science, Matsuyama, 2017, ISSFD-2017-014 / ISTS2017-d-014. 